\title{
A Novel Rotary Dual-Stage Actuator Positioner
}

\author{
Jinchuan Zheng, Aurélio Salton, and Minyue Fu
}

\begin{abstract}
This paper presents the design and control of a rotary dual-stage actuator (DSA) system, which consists of a voice coil motor (VCM) and a piezoelectric (PZT) microactuator. The performance of the mechanical design is analyzed using a finite element method (FEM) software, whose simulation results are validated experimentally. A nonlinear tracking control is applied to the developed DSA platform for reduced settling time and improved disturbance rejection. Experimental results demonstrate that the applied control design reduces the settling time by up to $50 \%$ when compared to a conventional design. Moreover, it is shown that the DSA system can achieve an improvement of $80 \%$ regarding disturbance rejection when compared to a single-stage actuator.
\end{abstract}

\section{INTRODUCTION}

A dual-stage actuator (DSA) is comprised of two actuators connected in series. The primary (coarse) actuator is characterized by a long travel range but poor accuracy and slow response time. The secondary (fine) actuator is of higher precision and faster response, but with limited travel range. An improved performance can be achieved by combining both actuators and making use of an appropriate control strategy, because the defects of one actuator may be compensated by the merits of the other. Different types of DSA are already seen in industrial applications. For example, the use of DSAs is popular in hard disk drives (HDDs), providing the system with a significant increase in the servo bandwidth and disturbance rejection, thus pushing the HDD track density [1], [2]. Other DSA systems include the dualstage machine tools [3], macro/micro robot manipulators [4], XY positioning tables [5], and wafers alignment in microlithography [6].

Motivated by the fact that some applications require a long range over a compact space (such as HDDs) and may benefit from rotary systems over translational ones, we have designed and manufactured the novel rotary DSA prototype seen in Fig. 1. While HDDs are a classical example, some larger scale systems that may benefit from a rotary dynamics include micro assembly stations [7], microscanners [8], surface-mount devices (SMD), among others. In order to satisfy the requirements of the above applications, we have designed our system to provide an accuracy of $\pm 0.15 \mu \mathrm{m}$ with a bandwidth of $200 \mathrm{~Hz}$, over a travel range of $73 \mathrm{~mm}$.

While designing our prototype, we faced the difficulty that few large scale rotary DSA systems existed, leaving us with few references to base the mechanical structure design on.

The authors are with the School of Electrical Engineering and Computer Science, The University of Newcastle, Callaghan, NSW 2308, Australia. This work was supported by the Australian Research Councils Center of Excellence for Complex Dynamic Systems and Control (CDSC). (e-mail: jinchuan.zheng@newcastle.edu.au; aurelio.salton@studentmail.newcastle.edu.au; minyue.fu@newcastle.edu.au).

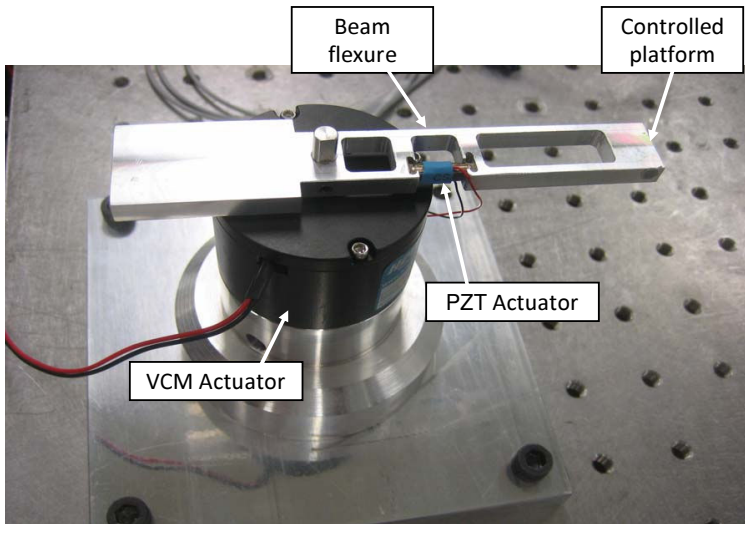

Fig. 1. A developed rotary DSA positioning system.

Furthermore, the structure of the system is too complex to be designed and analyzed analytically. In order to overcome these difficulties we have made use of a finite element method (FEM) computer software to aid our mechanical design. We will also demonstrate how to control the rotary DSA by adapting an exiting control law previously designed for a translational DSA.

The paper is organized as follows: Section II describes the system and the mechanical design, and presents the rotary DSA plant model; Section III exposes the control design; Section IV shows some experimental results; and Section V concludes the paper.

\section{MECHANISM DESIGN}

\section{A. System Description}

The developed rotary DSA is shown in Fig. 1. It comprises a voice coil motor (VCM) as the coarse actuator and a piezoelectric (PZT) microactuator as the fine one. Both actuators are assembled on a flexure beam, whose lateral displacement at the tip is the control objective. The VCM is positioned in the center of mass of the beam and is responsible for rotating the whole system, thus, providing it with a long travel range. The PZT actuator is placed in the middle of the beam and, by expanding and contracting, causes the flexure body to bend, which results in a limited but fast displacement at the tip of the beam.

The actuators are respectively driven by a VCM driver and a PZT amplifier. The VCM driver has a voltage input limit of $\pm 0.7 \mathrm{~V}$. The PZT actuator has a stroke limit of $13 \mu \mathrm{m}$ and the PZT amplifier has a voltage input limit of $10 \mathrm{~V}$. In the experimental setup, the tip position was measured by a laser Doppler Vibrometer (LDV) with an accuracy of $10 \mathrm{~nm}$. 


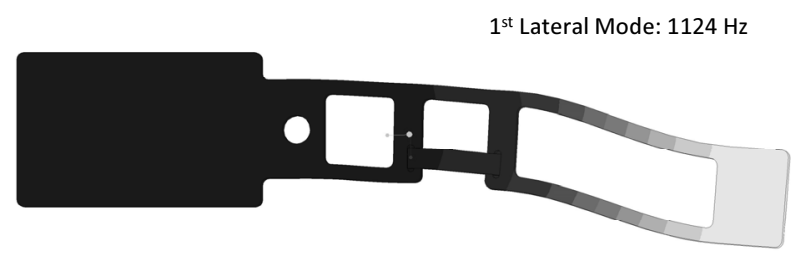

Fig. 2. The resonance mode shape of the lateral direction of the flexure beam $(1124 \mathrm{~Hz})$.

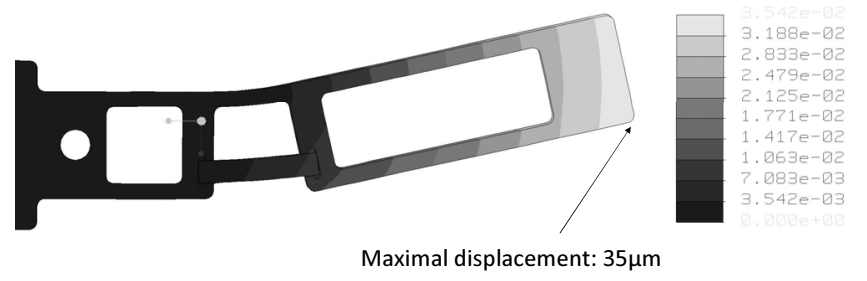

Fig. 3. Maximum displacement of the beam due to the PZT actuator enlarged deformation in order to illustrate the effect of the PZT.

\section{B. Flexure Beam Design and Analysis}

Several FEM simulations were performed in order to achieve the final structure seen in Fig. 1. Our main concerns were regarding the resonance frequency of the beam, the maximum displacement at the tip achieved by the PZT actuator and the stress distribution along the beam. The flexure beam material chosen was the commercial aluminum alloy AL5005 due to its reduced cost and satisfactory performance.

Fig. 2 shows the shape of the lateral directional resonant frequency of the rotary DSA. According to the simulation analysis, this frequency is $1124 \mathrm{~Hz}$, however, it won't be possible to drive the system at this bandwidth during implementation. Piezoelectric devices such as the one used in this application have the limitation that they are "push only" actuators (the actuator is unable to pull the structure to which it is attached). In other words, when the PZT contracts, the flexure beam must contract itself to the original position without any help from the actuator. It is crucial, therefore, that the acceleration of the flexure beam is equal to, or higher than, the acceleration of the driving input (the PZT actuator $)^{1}$. In order to achieve the desired acceleration of the moving part of the beam we must place a thin metal shim between the PZT and one of the walls in the cavity where it is placed. The effect of this metal shim is equivalent to that of pre-loading the flexure beam, which increases its acceleration during contraction. The amount of pre-load applied is proportional to the thickness of the metal shim used. Following the work presented in [9] - which shows a form of calculating the thickness of the metal shim in terms of the desired bandwidth of the actuator - we made use of a metal shim of $250 \mu \mathrm{m}$ thickness, so that our secondary actuator could be driven at frequencies higher than 1000 $\mathrm{Hz}^{2}$. Then, during the control implementation, we offset the PZT actuator to the middle of its travel range by applying

\footnotetext{
${ }^{1}$ If this is not the case the PZT will contract faster than the flexure moving part, resulting in separation between the beam and the actuator.

${ }^{2}$ The next simulations were performed considering the applied pre-load.
}

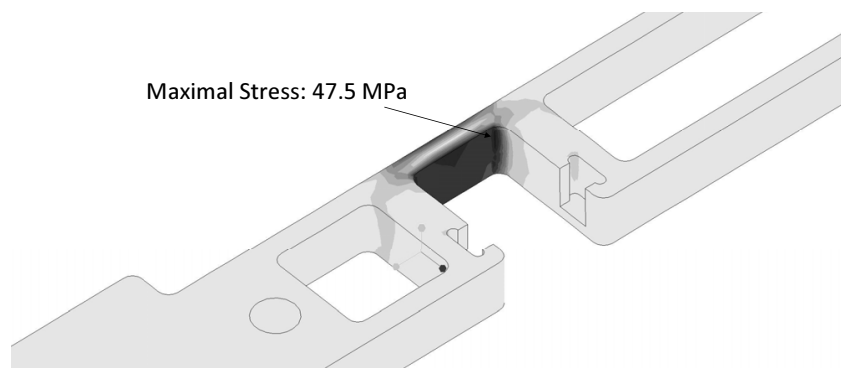

Fig. 4. Stress distribution along the structure of the beam.

a constant $5 \mathrm{~V}$ input, achieving a "push-pull" response from this actuator.

Fig. 3 shows the maximum displacement at the tip of the beam achieved by the PZT actuator, this figure presents an enlarged deformation of the beam in order to illustrate the effect of the PZT. Note that the structure of the beam allows the stroke limit of $13 \mu \mathrm{m}$ of the PZT to be amplified, causing a greater displacement at the tip. Considering the fact that the main limitation of the fine actuator is its travel range, it was of our interest to amplify the stroke limit of the PZT as much as possible. However, this became a trade off between displacement at the tip, resonance frequency and stress distribution on the beam. In order to accommodate these three parameters we had to limit the maximal displacement of the tip at $\pm 17 \mu \mathrm{m}$.

The next analysis to be performed was the stress distribution, showed in Fig. 4. This analysis was performed for the maximal static force applied by the PZT. The result shows that the maximum stress on the beam is $47.5 \mathrm{MPa}$, which is less than $50 \%$ of the Yield Strength of the material, showing that the risk of damaging the beam is small.

\section{Plant Modeling}

While building the DSA model the beam is idealized as the connection of two rigid bodies (arms) with lengths $l_{1}$ and $l_{2}$ and moments of inertia $J_{1}$ and $J_{2}$. As seen in Fig. 5 , the VCM (PZT) actuator rotates the primary (secondary) arm of the beam producing an angular displacement $\theta_{1}\left(\theta_{2}\right)$. The connection between both arms is idealized as a revolute joint with rotational spring constant $k_{2}$ and damping $c_{2}$. The arms have masses $m_{1}$ and $m_{2}$ and it was assumed a constant damping friction $c_{1}$ on the VCM joint.

The equations of motion of the DSA were derived using the Lagrangian method. From the Lagrangian equations

$$
\frac{d}{d t}\left(\frac{\partial L}{\partial \dot{\theta}_{i}}\right)-\left(\frac{\partial L}{\partial \theta_{i}}\right)=f_{i}, \quad i=1,2,
$$

where $L=K-P$ is the difference between the kinetic and potential energies of the system, and

$$
f_{i}=\operatorname{sat}\left(u_{i}\right)-c_{i} \dot{\theta}_{i}, \quad i=1,2 .
$$

The saturation function $\operatorname{sat}\left(u_{i}\right)$ for $i=1(\mathrm{VCM})$ and $i=2$ (PZT) is defined as:

$$
\operatorname{sat}\left(u_{i}\right)=\operatorname{sgn}\left(u_{i}\right) \min \left\{\bar{u}_{i},\left|u_{i}\right|\right\},
$$




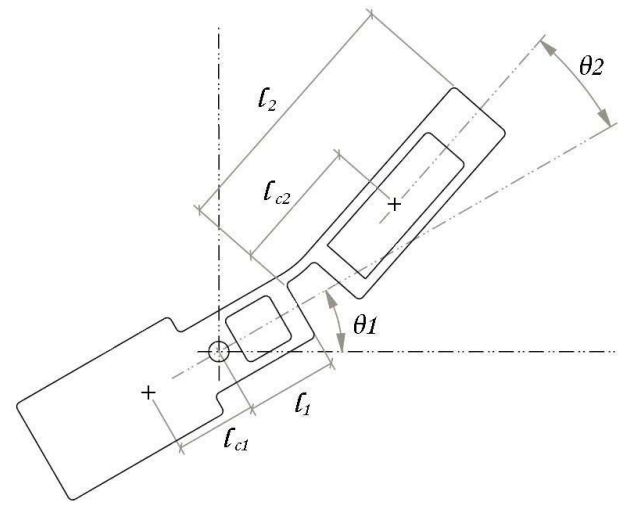

Fig. 5. Rotary dual-stage actuator mechanism.

where $\bar{u}_{i}$ is the saturation level of the $i$-th control input.

Solving equation (1) for $\theta_{1}$ and $\theta_{2}$, we obtain the equations of motion of the DSA:

$$
J\left(\theta_{2}\right) \ddot{\theta}+C\left(\theta_{2}, \dot{\theta}\right) \dot{\theta}+K \theta=u,
$$

where

$$
\theta=\left[\begin{array}{l}
\theta_{1} \\
\theta_{2}
\end{array}\right], \quad u=\left[\begin{array}{c}
\operatorname{sat}\left(u_{1}\right) \\
\operatorname{sat}\left(u_{2}\right)
\end{array}\right], \quad K=\left[\begin{array}{cc}
0 & 0 \\
0 & k_{2}
\end{array}\right] .
$$

Furthermore, the elements of the matrix $J\left(\theta_{2}\right)$ are:

$$
\begin{aligned}
J\left(\theta_{2}\right)_{11}= & m_{2}\left(l_{1}^{2}+l_{c 2}^{2}+2 l_{1} l_{c 2} \cos \theta_{2}\right) \\
& +J_{1}+J_{2}+m_{1} l_{c 1}^{2} \\
J\left(\theta_{2}\right)_{12}= & J_{2}+m_{2} l_{c 2}\left(l_{c 2}+l_{1} \cos \theta_{2}\right) \\
J_{22}= & J_{2}+m_{2} l_{c 2}^{2} \\
J\left(\theta_{2}\right)_{21}= & J\left(\theta_{2}\right)_{12}
\end{aligned}
$$

with $l_{c 2}$ representing the distance from the revolute joint to the center of mass of the second arm, and

$$
C\left(\theta_{2}, \dot{\theta}\right)=\left[\begin{array}{cc}
c_{1}-2 m_{2} l_{1} l_{c 2} \dot{\theta}_{2} \sin \theta_{2} & -m_{2} l_{1} l_{c 2} \dot{\theta}_{2} \sin \theta_{2} \\
m_{2} l_{1} l_{c 2} \dot{\theta}_{1} \sin \theta_{2} & c_{2}
\end{array}\right] .
$$

In this paper, the following simplifying assumptions were made:

1) The trigonometric functions were approximated by $\cos \theta_{2} \approx 1$ and $\sin \theta_{2} \approx \theta_{2}$

2) The system was represented by a decoupled dual-input single-output (DISO) system;

3) The primary system was modeled as a double integrator;

Assumption 1 is justified once the angular displacement $\theta_{2}$ is very small; assumption 2 holds due to the fact that the coupling force between the actuators is negligible; and assumption 3 was fulfilled by the design of a model-based friction compensator for the VCM actuator.

The system output is the arc displacement at the tip of the beam, hence, $y_{V}=\left(l_{1}+l_{2}\right) \theta_{1}, y_{P}=l_{2} \theta_{2}$ and the total output is given by $y=y_{V}+y_{P}$. In this way, we can treat the DSA as a linear decoupled dual-input single-output (DISO) system, which is represented in a state-space form as:

$$
\left\{\begin{array}{rlrl}
\Sigma_{1}: \dot{x}_{1} & =A_{1} x_{1}+B_{1} \operatorname{sat}\left(u_{1}\right), & & x_{1}(0)=0 \\
\Sigma_{2}: \dot{x}_{2} & =A_{2} x_{2}+B_{2} \operatorname{sat}\left(u_{2}\right), & & x_{2}(0)=0 \\
y & =y_{V}+y_{P}=C_{1} x_{1}+C_{2} x_{2}, &
\end{array}\right.
$$
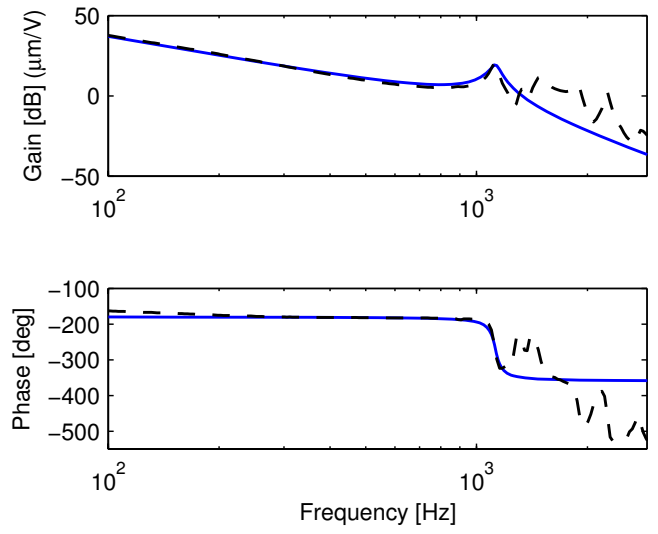

(a)
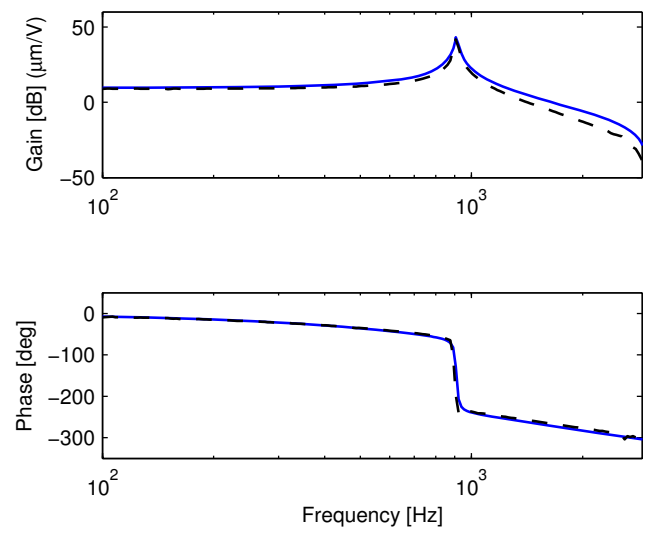

(b)

Fig. 6. Frequency responses of the DSA system (dashed line: measured response; solid line: simulated response). (a) VCM actuator. (b) PZT actuator.

where $x_{1}=\left[\begin{array}{ll}y_{V} & \dot{y}_{V}\end{array}\right]^{T}, x_{2}=\left[\begin{array}{ll}y_{P} & \dot{y}_{P}\end{array}\right]^{T}$, and

$$
\begin{array}{ll}
A_{1}=\left[\begin{array}{ll}
0 & 1 \\
0 & 0
\end{array}\right], & B_{1}=\left[\begin{array}{c}
0 \\
b_{1}
\end{array}\right], \quad C_{1}=\left[\begin{array}{ll}
1 & 0
\end{array}\right], \\
A_{2}=\left[\begin{array}{cc}
0 & 1 \\
a_{1} & a_{2}
\end{array}\right], & B_{2}=\left[\begin{array}{c}
0 \\
b_{2}
\end{array}\right], \quad C_{2}=\left[\begin{array}{ll}
1 & 0
\end{array}\right] .
\end{array}
$$

With $b_{1}=\left(l_{1}+l_{2}\right) / J_{11}, a_{1}=-c_{2} / J_{22}, a_{2}=-k_{2} / J_{22}$ and $b_{2}=l_{2} / J_{22}$.

The DSA model parameters were identified to match the measured frequency responses of the plant. The VCM and PZT actuators frequency responses were measured separately and are shown in Fig. 6. It can be seen that their resonance frequencies are at $1170 \mathrm{~Hz}$ and $900 \mathrm{~Hz}$, respectively. These results show good agreement with the FEM results. We used digital notch filters to actively compensate the resonances.

The nonlinearity seen in the VCM plot shows the friction acting in this actuator. Here, we employ a model-based control structure similar to the one in [10] and [11] to compensate for unwanted friction and disturbances in this actuator. From the low frequency response of the PZT actuator one can see that there exists some level of interaction between 
the PZT and VCM actuator, however, further experimental results have showed that this coupling is very small and could be neglected.

The identified DSA model parameters are:

$$
\begin{array}{lll}
a_{1}=-5.04 \times 10^{7}, & b_{1}=2.00 \times 10^{7}, & \bar{u}_{1}=0.5 \mathrm{~V}, \\
a_{2}=7.10 \times 10^{3}, & b_{2}=1.61 \times 10^{8}, & \bar{u}_{2}=3.0 \mathrm{~V} .
\end{array}
$$

From now on, we will consider the DSA as in (5) and use this linear decoupled model for the control design.

\section{CONTROL DESIGN}

Once our system is described by the linear de-coupled model in (5) and resembles the translational DSA in [11], we have chosen to adapt the control strategy seen in [11] and [12] with the purpose of demonstrating that we can effectively control the rotary DSA. Recall that the control objective is to enable the total position output $y$ to track a step command input of amplitude $y_{r}$ rapidly with no overshoot larger than $0.15 \mu \mathrm{m}$.

In order to achieve the control objective the VCM and PZT control laws were designed separately. First, a near timeoptimal control law is designed to yield the VCM loop with a small damping ratio, allowing some level of overshoot and providing the system with a fast rise time. Then, a composite nonlinear control law is designed for the PZT actuator so that it compensates for the overshoot caused by the VCM. Notice that it is crucial that the VCM overshoots within the travel range of the PZT actuator, so that the latter may compensate for it.

Since the head position $y$ is the only measurable signal for feedback control, we need to estimate the VCM and PZT actuator states by using the identified DSA model. The state estimator was designed using pole placement method by selecting the well damped estimator poles to be two to six times faster than the DSA servo bandwidth. Recall that, due to the separation principle [13], the design of the control laws $u_{1}$ and $u_{2}$ and the design of the estimator can be performed separately, even though they are implemented together. Therefore, we will present the control law design assuming that the true states are available for feedback, however, the estimated states will be used during the controller implementation.

\section{A. VCM Actuator Control Design}

For the VCM actuator we have applied the well known proximate time-optimal servomechanism (PTOS) control [14]. This is a near time-optimal control strategy that can accommodate plant uncertainty, measurement noise and actuator saturation. The PTOS control law uses the maximal acceleration provided by the actuator (saturates the actuator) when the system output is far from the reference; as the output approaches the reference point it introduces a linear region, reducing the control chatter. In this linear region we have designed a linear state feedback law $u_{1}=K x_{1}$ via pole placement. By designing the gain $K$ via pole placement we are able to assign the damping ratio of the closed-loop system and, thus, the amount of overshoot. This is crucial once we must limit the VCM actuator overshoot to be within the travel range of the PZT actuator. A parameterization of the gain $K$ in terms of the desired bandwidth and damping ratio is given by:

$$
K=\frac{1}{b_{1}}\left[\begin{array}{ll}
4 \pi^{2} f_{1}^{2} & 4 \pi f_{1} \zeta_{1}
\end{array}\right]
$$

where $\zeta_{1}$ and $f_{1}$ (in hertz) represent, respectively, the damping ratio and the undamped natural frequency of the closedloop system $C_{1}\left(s I-A_{1}+B_{1} K\right)^{-1} B_{1}$, whose poles are placed at $2 \pi f_{1}\left(\zeta_{1} \pm j \sqrt{1-\zeta_{1}^{2}}\right)$.

\section{B. PZT Actuator Control Design}

The design of the PZT actuator is divided in two parts: a linear feedback gain, which guarantees the global asymptotical stability of the closed-loop system, and a nonlinear control law, which enables the PZT to compensate for the overshoot generated by the VCM actuator. Both designs are then combined to form a composite nonlinear controller for the PZT actuator. We shall present the linear and the nonlinear feedback design separately.

\section{Linear Feedback: Design a linear feedback control law}

$$
u_{2 L}=W x_{2}
$$

where $W=\left[\begin{array}{ll}w_{1} & w_{2}\end{array}\right]$ is chosen such that the PZT actuator control system given by

$$
\dot{x}_{2}=A_{2} x_{2}+B_{2} \operatorname{sat}\left(W x_{2}\right)
$$

is globally asymptotically stable (GAS) and the corresponding closed-loop system, in the absence of input saturation, $C_{2}\left(s I-A_{2}+B_{2} W\right)^{-1} B_{2}$ has a higher damping ratio and a higher undamped natural frequency than those of the VCM actuator control loop.

Nonlinear Feedback: Construct the nonlinear feedback control law

$$
\begin{aligned}
& u_{2 N}=\gamma\left(y_{r}, y\right) H\left[\begin{array}{c}
y_{V}-y_{r} \\
\dot{y}_{V}
\end{array}\right] \\
& H=\frac{1}{b_{2}}\left[\begin{array}{ll}
\left(a_{1}+b_{2} w_{1}+b_{1} k_{1}\right) & \left(a_{2}+b_{2} w_{2}+b_{1} k_{2}\right)
\end{array}\right]
\end{aligned}
$$

where the choice of $H$ and $\gamma\left(y_{r}, y\right)$ will be clear latter. For now it suffices to know that $H$ is taken to achieve desired closed-loop system dynamics and $\gamma\left(y_{r}, y\right)$ is any nonnegative function, locally Lipschitz in $y$, chosen to enable the PZT actuator to reduce the overshoot caused by the VCM.

Composite Nonlinear Control: Combine the linear and nonlinear feedback laws.

$$
\begin{aligned}
u_{2} & =u_{2 L}+u_{2 N} \\
& =W x_{2}+\gamma\left(y_{r}, y\right) H\left[\begin{array}{c}
y_{V}-y_{r} \\
\dot{y}_{V}
\end{array}\right] .
\end{aligned}
$$

If we consider the DSA system in (5) with the VCM actuator $\sum_{1}$ under the PTOS control law, and the PZT actuator $\Sigma_{2}$ under the nonlinear control law (11), then it can be shown that this composite control law will drive the tip 
position $y$ to asymptotically track any step command input of amplitude $y_{r}$ [11].

The value of $\gamma\left(y, y_{r}\right)$ does not affect the ability of the overall DSA closed-loop system to track asymptotically any step command input. However, an appropriate choice of $\gamma\left(y, y_{r}\right)$ can be utilized to improve the transient performance of the overall closed-loop system ${ }^{3}$. The DSA closed-loop system dynamics change from the VCM control loop to the PZT control loop as $\gamma$ increases from 0 to 1 . This transition is smooth due to $\gamma$, and it is from the VCM loop to the PZT loop due to the proper choice of $H$. In order to see this, notice that when $\gamma$ changes from 0 to 1 the zeros of the DSA closed loop are moved from the pole location of the PZT control loop to those of the VCM control loop. Since the zeros near the poles reduce the effects of the poles on the total response, we can use $\gamma$ to tune the system dynamics for desired performance. In other words, the composite nonlinear control law is used to make the DSA closed loop highly damped as the output $y$ approaches the reference, compensating for the overshoot caused by the VCM.

Therefore, one may conclude that a good choice of the function $\gamma$ would come from making it a function of the tracking error $\left(y_{r}-y\right)$. For the purpose of this application

$$
\gamma\left(y_{r}, y\right)=e^{-\beta\left|y_{r}-y\right|},
$$

where $\beta \geq 0$ is a tuning parameter. This function changes from 0 to 1 as $y \rightarrow y_{r}$. The parameter $\beta$ can be adjusted with respect to the amplitude of $y_{r}$ and describes how fast should $\gamma$ increase, i.e., how fast should the transition from the VCM loop to the PZT loop occur.

\section{EXPERIMENTAL RESULTS}

The proposed DSA tracking control has been applied to the DSA seen in Fig. 1. The controller was implemented in a DSP system (dSPACE 1103) with a sampling rate of 20 $\mathrm{kHz}$.

For the VCM control loop we have chosen $f_{1}=20 \mathrm{~Hz}$, which means that the PTOS controller works in its linear region for step references of amplitude smaller than $633 \mu \mathrm{m}$. In this linear region we must still calculate the desired value of $\zeta_{1}$. Since the overshoot should be limited to be within the PZT travel range, we must calculate $\zeta_{1}$ and $K$ online as a function of $y_{r}$. The experimental results showed that the actual maximal displacement at the tip achieved by the PZT actuator was of $20 \mu \mathrm{m}$. Since the overall response is dominated by the PZT loop when $\left|y_{r}\right| \leq 10 \mu \mathrm{m}$, we can set $\zeta_{1}$ as any constant less than 1 in this region. However, when $\left|y_{r}\right|>10 \mu \mathrm{m}$ the value of $\zeta_{1}$ must be numerically derived so that the overshoot of the VCM actuator is within $\pm 10 \mu \mathrm{m}$. Hence,

$$
\zeta_{1}\left(y_{r}\right)= \begin{cases}0.3, & y_{r} \leq 10 \mu m, \\ \left|\frac{\ln (10)-\ln \left(y_{r}\right)}{\sqrt{\pi^{2}+\left(\ln (10)-\ln \left(y_{r}\right)\right)^{2}}}\right|, & y_{r}>10 \mu m .\end{cases}
$$

${ }^{3}$ For a detailed discussion on the choice of $\gamma\left(y, y_{r}\right)$ please refer to [11] and [12].

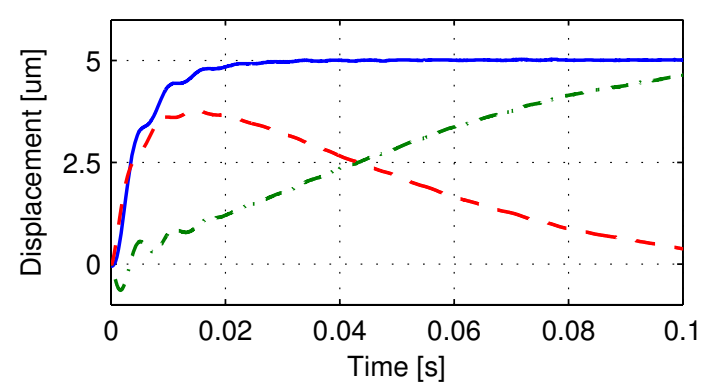

(a)

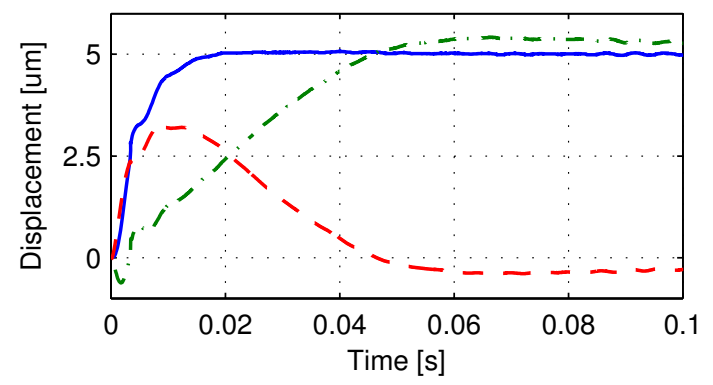

(b)

Fig. 7. Seeking responses to $y_{r}=5 \mu \mathrm{m}$. The $27 \mathrm{~ms}$ settling time achieved by the conventional controller is reduced to $14 \mathrm{~ms}$ by the proposed control design (solid lines: system output; dashed line: PZT; dash-dotted line: VCM). (a) Conventional DSA control. (b) Proposed DSA control.

And the linear gain $K$ is given by

$$
K=10^{-5} \times\left[\begin{array}{lll}
78.96 & 1.26 \zeta_{1}\left(y_{r}\right)
\end{array}\right] .
$$

For the PZT control design we have

$$
W=\left[\begin{array}{ll}
3.027 \times 10^{-1} & 3 \times 10^{-5}
\end{array}\right],
$$

resulting from $f_{2}=200 \mathrm{~Hz}$ and $\zeta_{2}=0.9$. The nonlinear feedback gain $H$ is given by:

$$
H=\left[-9.69 \times 10^{-3}-1.41 \times 10^{-5} \zeta_{1}\left(y_{r}\right)\right] .
$$

We would also like to compare our proposed controller with a conventional form of DSA control. In order to do so, we have fixed the value of $\zeta_{1}$ such that the primary actuator presents no overshoot. During the step responses, we define the settling time to be the time that it takes for the total position output $y$ to enter and remain within $\pm 0.15 \mu \mathrm{m}$ relative to the setpoint.

\section{A. Step Responses}

Here we present the step responses obtained for reference steps of $5 \mu \mathrm{m}$ and $10 \mu \mathrm{m}$. The results in Fig. 7 show the step responses of both the conventional and the proposed DSA controller for a reference amplitude of $5 \mu \mathrm{m}$. One can see that, when no overshoot is allowed, the conventional controller presents a settling time of $27 \mathrm{~ms}$, while the proposed control law achieves a settling time of $14 \mathrm{~ms}$. Fig. 8 shows the responses for a $10 \mu \mathrm{m}$ reference step. The conventional controller has a settling time of $36 \mathrm{~ms}$ while the proposed control law achieves a settling time of $18 \mathrm{~ms}$. These results show that the proposed design significantly reduced 


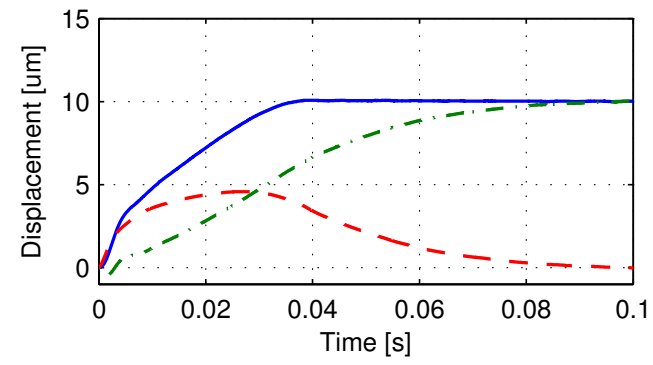

(a)

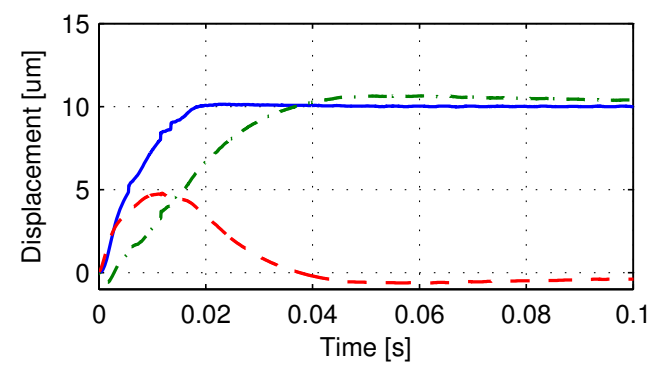

(b)

Fig. 8. Seeking responses to $y_{r}=10 \mu \mathrm{m}$. The settling time of $36 \mathrm{~ms}$ achieved by the conventional controller is reduced to $18 \mathrm{~ms}$ by the proposed control design (solid lines: system output; dashed line: PZT; dash-dotted line: VCM). (a) Conventional DSA control. (b) Proposed DSA control.

the settling time when compared to the conventional one (an improvement of up to $50 \%$ was achieved).

\section{B. Disturbance Rejection}

We have also tested the DSA system ability to reject disturbance. The disturbance signal consists of three sinusoidal components with frequencies $5 \mathrm{~Hz}, 15 \mathrm{~Hz}$ and $30 \mathrm{~Hz}$, as in the top plot of Fig. 9. The signals are artificially generated by the DSP and directly added onto the VCM control input. The middle plot of Fig. 9 shows the output response when the PZT actuator is turned off. One can see that the position error is within $\pm 6 \mu \mathrm{m}$. On the other hand, when the PZT is turned on, the position error falls to $\pm 1 \mu \mathrm{m}$, as seen in the bottom plot. These results show an improvement of over $80 \%$ regarding disturbance rejection during track following.

\section{CONCLUSIONS AND FUTURE WORKS}

We have proposed a design and control methodology for a rotary DSA system. Due to the complexity of the structure, the mechanical design of the system was performed with the aid of the FEM software, which served as a guide for the structure of the beam to be created. The nonlinear control design method (adapted from a translational DSA) proved itself to be an efficient form of control for the rotary DSA. The experimental results showed that an improvement of over $50 \%$ in reducing the settling time and $80 \%$ in tracking error reduction is achieved when choosing the DSA structure over the conventional single-stage one. Our future work will include the model nonlinearities and the interaction between actuators in order to improve the bandwidth of the system.
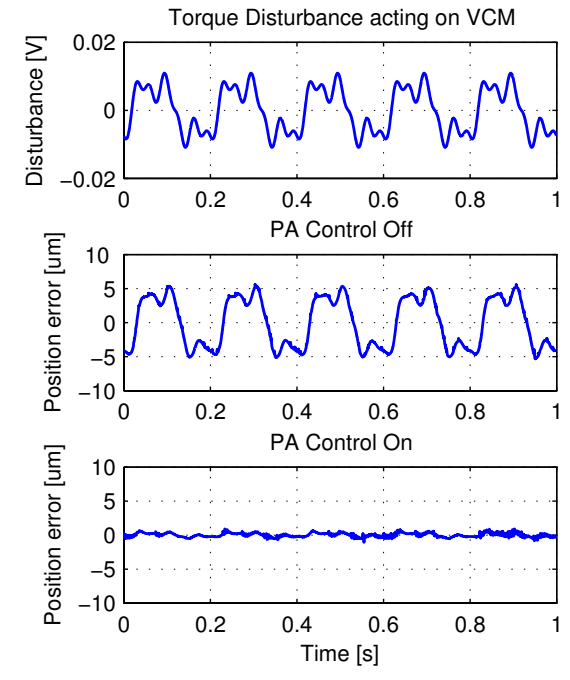

Fig. 9. Steady-state position error under disturbance input. When PA control is switched off, the position error under the VCM control loop is within $\pm 6 \mu \mathrm{m}$, while with PA control on, the position error is retained within $\pm 1 \mu \mathrm{m}$.

\section{REFERENCES}

[1] K. Mori, T. Munemoto, H. Otsuki, Y. Yamaguchi, and K. Akagi, “A dual-stage magnetic disk drive actuator using a piezoelectric device for a high track density," IEEE Trans. Magn., vol. 27, no. 6, pp. 52985300, Nov. 1991.

[2] L. Guo, D. Martin, and D. Brunnett, "Dual-stage actuator servo control for high density disk drives," in Proc. IEEE/ASME Int. Conf. Advanced Intelligent Mechatronics, 1999, pp. 132-137.

[3] B. Kim, J. Li, and T. Tsao, "Two-parameter robust repetitive control with application to a novel dual-stage actuator for noncircular machining," IEEE/ASME Trans. Mechatron., vol. 9, no. 4, pp. 644-652, Dec. 2004.

[4] A. Sharon, N. Hogan, and D. Hardt, "The macro/micro manipulator: an improved architecture for robot control," J. Robotics and computerintegrated manufacturing, vol. 10, pp.209-222, 1993.

[5] A. Elfizy, G. Bone, and M. Elbestawi, "Design and control of a dualstage feed drive," Int. J. Machine Tools Manuf., vol. 45, pp. 153-165, 2005.

[6] C. Lee and S. Kim, "An ultraprecision stage for alignment of wafers in advanced microlithography," Precision Engineering, vol. 21, pp. 113-122, 1997.

[7] N. Kawahara, T. Suto, T. Hirano, Y. Ishikawa, T. Kitahara, N. Ooyama, and T. Ataka, "Microfactories; new applications of micromachine technology to the manufacture of small products," Microsystem Technologies, vol. 3, no. 2, pp. 37-41, 1997.

[8] H. Cho and C. Ahn, "Magnetically-driven bi-directional optical microscanner," J. Micromech. Microeng., vol. 13, no. 3, pp. 383-389, 2003.

[9] D. Lee and D. Gweon, "Pseudo-resonant effect on a flexure-guided nano-positioning system," J. Korean Phys. Soc., vol. 48, no. 3, pp. 363-370, Mar. 2006.

[10] H. Choi, B. Kim, I. Suh, and W. Chung, "Design and robust highspeed motion controller for a plant with actuator saturation," J. Dyn. Sys., Meas., Control, vol. 122, pp. 535-541, Sep. 2000.

[11] J. Zheng and M. Fu, "Nonlinear feedback control of a dual-stage actuator system for reduced settling time," IEEE Trans. Contr. Syst. Technol., vol. 16, no. 4, pp. 717-725, Jul. 2008.

[12] J. Zheng, M. Fu, Y. Wang, and C. Du, "Nonlinear tracking control for a hard disk drive dual-stage actuator system," IEEE/ASME Trans. Mechatron., vol. 13, no. 5, pp. 510-518, Oct. 2008.

[13] G. F. Franklin, J. D. Powell, and A. Emami-Naeini, Feedback Control of Dynamic Systems, 3rd ed., Reading, MA: Addison-Wesley, 1994.

[14] M. Workman, "Adaptive Proximate Time-Optimal Control Servomechanisms," Ph.D. thesis, Stanford University, 1987. 